\title{
Strong electron heating and non-Maxwellian behavior in magnetic reconnection
}

\author{
M. Hoshino ${ }^{1}$, K. Hiraide ${ }^{1}$, and T. Mukai ${ }^{2}$ \\ ${ }^{1}$ University of Tokyo, 7-3-1 Hongo, Bunkyo-ku, Tokyo 113-0033, Japan \\ ${ }^{2}$ ISAS, 3-1-1 Yoshinodai, Sagamihara, Kanagawa 229-8510, Japan \\ (Received July 5, 2000; Revised November 29, 2000; Accepted December 1, 2000)
}

\begin{abstract}
We discuss the electron heating in the course of magnetic reconnection by using both the Geotail observation and the particle-in-cell simulation. Geotail observes several unique non-Maxwellian velocity distribution functions during the plasma sheet crossing in association with a fast plasma flow. We find that the observed distributions can be classified into four different types depending on the position in the plasma sheet. In the boundary between the lobe and the plasma sheet, the distribution consists of the cold plasma flowing toward the X-type region and the hot plasma escaping from the X-type region along the magnetic field. In the plasma sheet side of the boundary, the distribution becomes bi-Maxwellian distribution with $T_{\|}>T_{\perp}$. Inside the plasma sheet, the distribution is deformed into a hot and isotropic distribution. We discuss the physical mechanism responsible for those electron heating in a thin plasma sheet by using the kinetic reconnection simulation. We find that the dawn-dusk reconnection electric field as well as the turbulent waves excited by the strong Hall electric currents play an important role on the strong electron heating and acceleration.
\end{abstract}

\section{Introduction}

The plasma heating and acceleration is a long standing problem in space. Over the last several decades, considerable effort has been devoted toward understanding the basic mechanism of the plasma energization, and various mechanisms have been discussed. Magnetic reconnection is one of the important processes for the plasma heating in the plasma sheet, but our understanding on the plasma heating/acceleration process in reconnection is still poor.

In magnetohydrodyanamic (MHD) framework, Petschek reconnection with a pair of slow shocks (Petschek, 1964) can explain a rapid energy conversion from the magnetic field energy stored in the plasma sheet to the plasma kinetic energy, and the process has been extensively discussed by using the MHD simulations so far. This kind of reconnection process is widely applied for the explosive energy release phenomena such as solar flares and magnetospheric substorms. Although the reconnection studies in the MHD framework seem to be successful to understand the global dynamics in many applications, kinetic aspects beyond the MHD framework seem to be necessary to understand the reconnection in details, because reconnections observed in the Earth's magnetotail (e.g., Nishida, 2000) and in the laboratory plasmas (e.g., Yamada et al., 2001) are not simply understood by the conventional reconnection in MHD framework.

Such unresolved issues, for example, are as follows: 1) The slow shocks have been identified by the in site satellite observations in the magnetotail (Feldman et al., 1987; Saito et al., 1996), but the boundary between the lobe and

Copy right (c) The Society of Geomagnetism and Earth, Planetary and Space Sciences (SGEPSS); The Seismological Society of Japan; The Volcanological Society of Japan; The Geodetic Society of Japan; The Japanese Society for Planetary Sciences. the plasma sheet does not necessarily satisfy the slow mode shock jump condition even if the fast plasma flows are observed in the plasma sheet side (Saito et al., 1996). The conventional slow shock might not be the dominant process of the plasma heating in the Earth's magnetotail. 2) Non-Maxwellian plasma distribution functions are often observed in the magnetotail, and the time scale of plasma thermalization is thought to be not necessarily shorter than the dynamic time scale of reconnection. 3) Ion temperature is in general higher than electron temperature in the Earth's plasma sheet, and the differential plasma heating process is occurring. 4) Moreover, non-thermal high-energy ions and electrons over $\sim \mathrm{MeV}$ are often observed in association with reconnection (e.g., Terasawa and Nishida, 1976; Baker and Stone, 1977). It is required to understand reconnection physics beyond the MHD framework.

Based on the recent satellite observations and the kinetic simulation studies of reconnection, it is revealed that the thickness of the plasma sheet near the X-type region becomes of the order of ion skin depth (e.g., Hoshino et al., 1998; Hesse et al., 1999; Shay et al., 1999). Since the kinetic features beyond the MHD description appears in the macroscopic scale length, the self-consistent understanding of both the macroscopic evolution of reconnection and the microscopic behavior of plasma dynamics becomes important. In this paper, we study the electron heating by using the Geotail satellite observation and the particle-in-cell simulation, and discuss how and where the electrons are thermalized.

\section{Geotail Observation}

Figure 1 shows one of the hot electron events observed by Geotail. This data is taken in the magnetotail at a distance of about $24 R_{\mathrm{E}}$ from the Earth on December 10, 1996. 


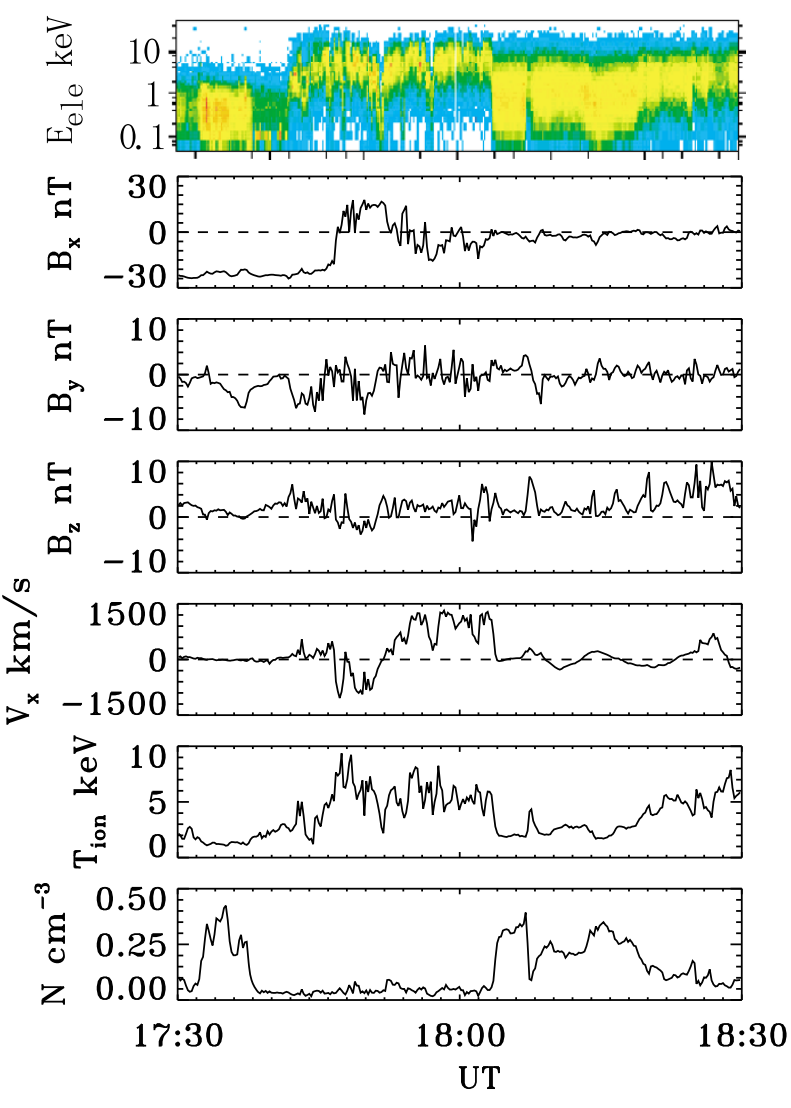

Fig. 1. Plasma and magnetic field measurements taken by the Geotail satellite are displayed for the December 10, 1996, reconnection event. From the top, the electron omni-directional energy spectrogram, the $x$, $y$, and $z$-component of the magnetic field, the plasma velocity $V_{x}$, the ion temperature, and the plasma density.

From the top, the electron omni-directional energy spectrogram, three components of the magnetic field, the ion flow velocity in the $x$-component, the ion temperature, and the plasma density. Geotail observes a plasma flow transition from tailward to earthward at $1752 \mathrm{UT}$, and during the fast flow region the weak magnetic field and the hot ion plasma are observed. This is suggestive that the satellite observes the magnetic reconnection region by moving relative to an $\mathrm{X}$-type neutral line. In the time interval from $1742 \mathrm{UT}$ to 1804 UT, we find the hot electrons from the electron energy spectrogram, while before 1742 UT and after 1804 UT the energy spectrograms show more or less the typical plasma sheet electron of several hundred $\mathrm{eV}$.

In the hot electron region from 1742 UT to $1804 \mathrm{UT}$, we observe non-Maxwellian electron distribution functions in addition to a standard Maxwellian-like distribution. Figure 2 shows four typical distribution functions observed in the hot electron region. The top four panels show a slice of threedimensional distribution function in a plane including the magnetic field direction and the $E \times B$ convection direction, measured by the Low Energy Particle (LPE) detector onboard Geotail (Mukai et al., 1994). The bottom panel shows one-dimensional cut along the magnetic field direction. The bottom time indicates the observation time for each distribution function. From the left, 1) "Maxwellian-like" distribution, 2) "truncated-cone" distribution where the phase space density in the lower energy region is almost flat, 3) "football" distribution where the temperature along the magnetic field line is higher than that of the perpendicular direction, and 4) "shifted-football" distribution that is similar to the football distribution, but the counter streaming flows of the cold and hot components coexist. Note that the hot "Maxwellian-like", "truncated-cone", and "football" distribution functions often contain the suprathermal population in the high energy range.

We have analyzed other data when Geotail traverses the plasma sheet during the fast plasma flow. For the hot electron events with $>$ a few $\mathrm{keV}$ temperature, we have confirmed that the electron distribution functions are more or less classified into the above four types. On the other hand, for the standard plasma sheet with the electron temperature of several hundred $\mathrm{eV}$, the classification into the above four classes becomes difficult, because the shoulder energy of the truncated-cone/football distribution is shifted to the lower energy with a large phase space density. Note that occurrence frequency of the hot electron event is rare.

It is interesting to study where and when those distribution functions are observed. We study the statistical property of the non-Maxwellian electron distributions. The data periods in our current analysis include January and February 1994, and December 1996. Figure 3 shows the relationship of the ion bulk flow velocity $V_{x}$ and the magnetic field $B_{x}$ for the distributions of "football", "shifted-football", and "truncated-core". The "football" distribution is shown in the closed circle, the "shifted-football" distribution is the open circle, and the "truncated-cone" is the open diamond. The magnetic field $B_{x}$ is normalized by the lobe magnetic field $B_{\text {lobe }}$ that can be estimated by assuming the pressure balance between the lobe and the plasma sheet, namely $B_{\text {lobe }}^{2} / 8 \pi=$ $B_{x}^{2} / 8 \pi+P$ where $P$ is the plasma gas pressure. In this statistical analysis, we remove ambiguous distributions that cannot be easily classified into the football, the shifted-football, or the truncated-cone.

From the diagram in Fig. 3, we find the "shifted-football" is observed only when the flow velocity is slow and the normalized magnetic field $B_{x} / B_{\text {lobe }}$ is almost unity. We also confirmed that this distribution is associated with the PSBL ion beams (e.g., Richerdson et al., 1987). Therefore we conclude that the "shifted-football" is observed in the boundary between the lobe and the plasma sheet. We further found that the cold electron population in the shifted-football is flowing in the opposite direction against the PSBL ion beams (Fujimoto et al., 1997; Nagai et al., 2001).

The "football" type is observed in the region with the intermediate magnetic field strength between the lobe and the neutral sheet. The football distribution can be observed in both fast and slow plasma flows. The "truncated-cone" type is often observed in the plasma sheet in association with the fast plasma flow. By comparing the domain of "football" and "truncated-cone" in the $v_{x}-B_{x}$ plane, we find that the "truncated-cone" distributions are located in the inner plasma sheet region.

The Maxwell distributions are basically observed in any region. In general, the cold Maxwellian is observed in the lobe side, and the hot Maxwellian is found in the plasma sheet. The very hot Maxwellian shown in Fig. 2 can be ob- 

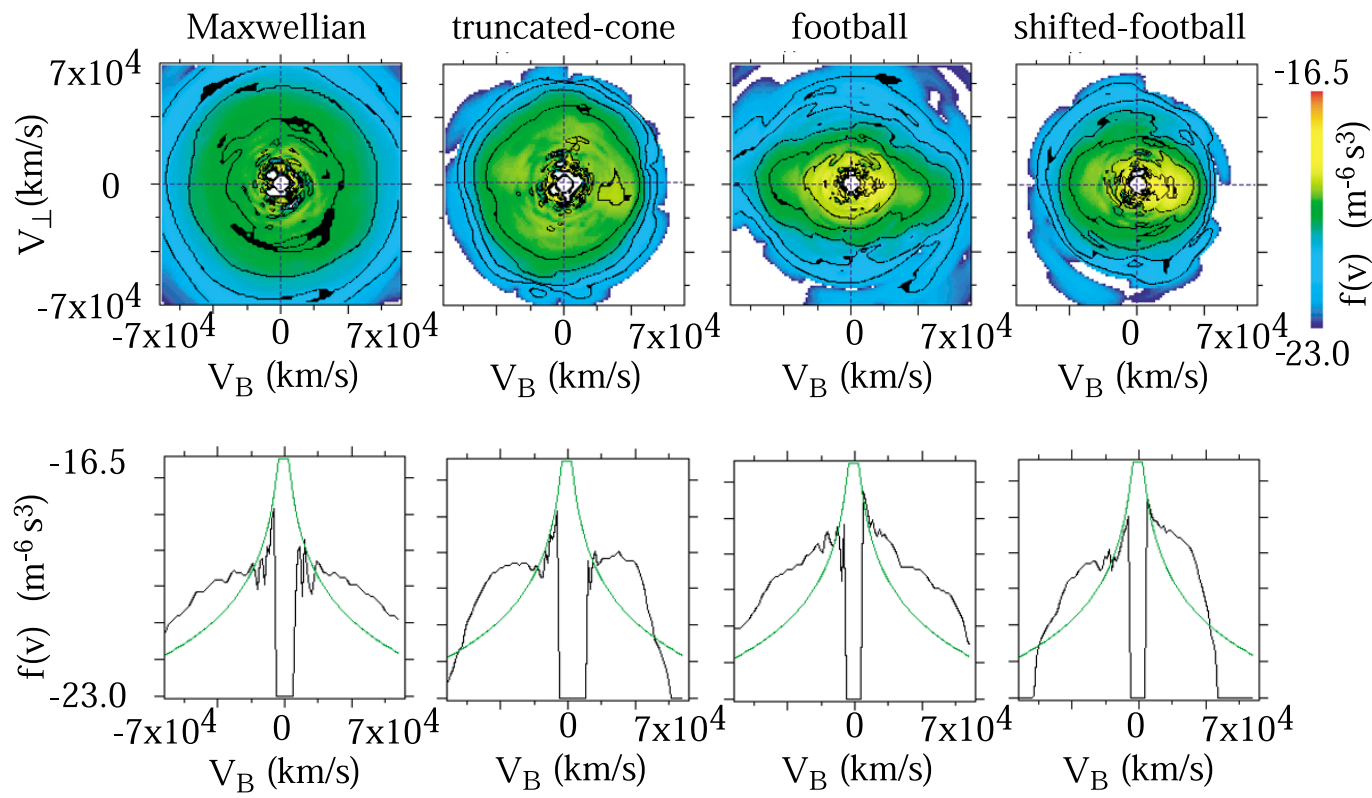

1756:19-1756:31 1749:03-1749:15 1746:01-1746:13 1750:40-1750:52

Fig. 2. Four typical electron velocity distribution functions are displayer for the save event as in Fig. 1. The time intervals for each distribution functions are indicated in the bottom. The top panels are the cut of the three-dimensional distribution functions that are shown in the reference coordinate system to the magnetic field. $V_{B}$ and $v_{\perp}$ correspond to the magnetic field direction and the plasma convection flow direction, respectively. The center of the cut is the origin of the spacecraft frame. The color scale show the level of the phase space density from $10^{-23}$ to $10^{-16.5} \mathrm{~s}^{3} \mathrm{~m}^{-6}$. The bottom panels are the slice of the distribution function along the magnetic field direction. The green lines show the one-count level for the LEP particle detector.

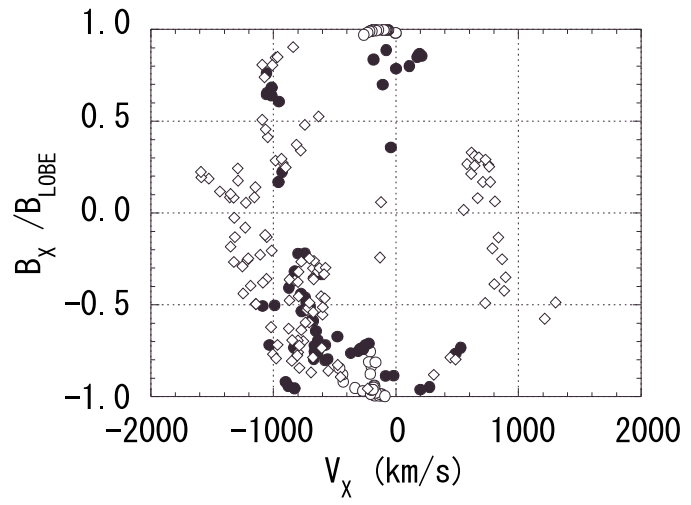

Fig. 3. The distributions in the $V_{x}-B_{x}$ plane for three typical velocity distribution functions: the shifted-football (open circles), the football (closed circles) and the truncated-cone (open diamonds).

served in association with the fast plasma flow and in the inner plasma sheet.

Another important point we found from the Geotail data is the non-gyrotropic nature of the ion distribution function in association with the above non-Maxwellian electrons (not shown here). The behavior of the non-gyrotropic ions has been studied as a function of the distance from the X-type neutral line in a thin plasma sheet (Hoshino, 1998). Since we found that some of the non-gyrotropic ions observed by Geotail can be understood as the behavior of magnetic reconnection region, we think that the above non-Maxwellian electrons are observed in the thin plasma sheet during magnetic reconnection. It is also known that the thickness of the plasma sheet becomes of the order of ion skin depth dur- ing magnetic reconnection from the kinetic simulation study (e.g., Hoshino et al., 1998; Hesse et al., 1999; Shay et al., 1999).

In the following sections, motivated by the above observations, we discuss how the strong electron heating is occurring in the course of magnetic reconnection by using the particle-in-cell simulation. We think that the Hall current which plays an important role in a thin plasma sheet is responsible to the electron heating. In a thin plasma sheet, the magnitude of the Hall current could become of the order of the dawn-dusk electric current, and then strong waves would be generated by utilizing the free energy of the strong Hall current, which in turn will thermalize the electrons.

\section{Numerical Simulation}

We use the two-dimensional, particle-in-cell simulation to study the behavior of electron dynamics. We assume the Harris equilibrium as the initial condition, where the electric current flows only in the y direction and no $B_{z}$ magnetic field component exists (Harris, 1962). The initial hot plasma localized inside the plasma sheet balances the magnetic pressure produced self-consistently by the electric current. However, we added the low-density, cold plasma in the lobe region to demonstrate the Earth's magnetotail situation. We also drive a localized external electric field outside the plasma sheet to initiate the reconnection in the center of the simulation box (Hoshino, 1991). The initial set up is almost same as our previous study by Hoshino et al. (1998).

In our current study, we simulate the nonlinear evolution of magnetic reconnection in much high spatial resolution in order to resolve the electron inertia scale. The physical system size is $L_{x} / \lambda=40$ and $L_{z} / \lambda=16$, where $\lambda$ is the 

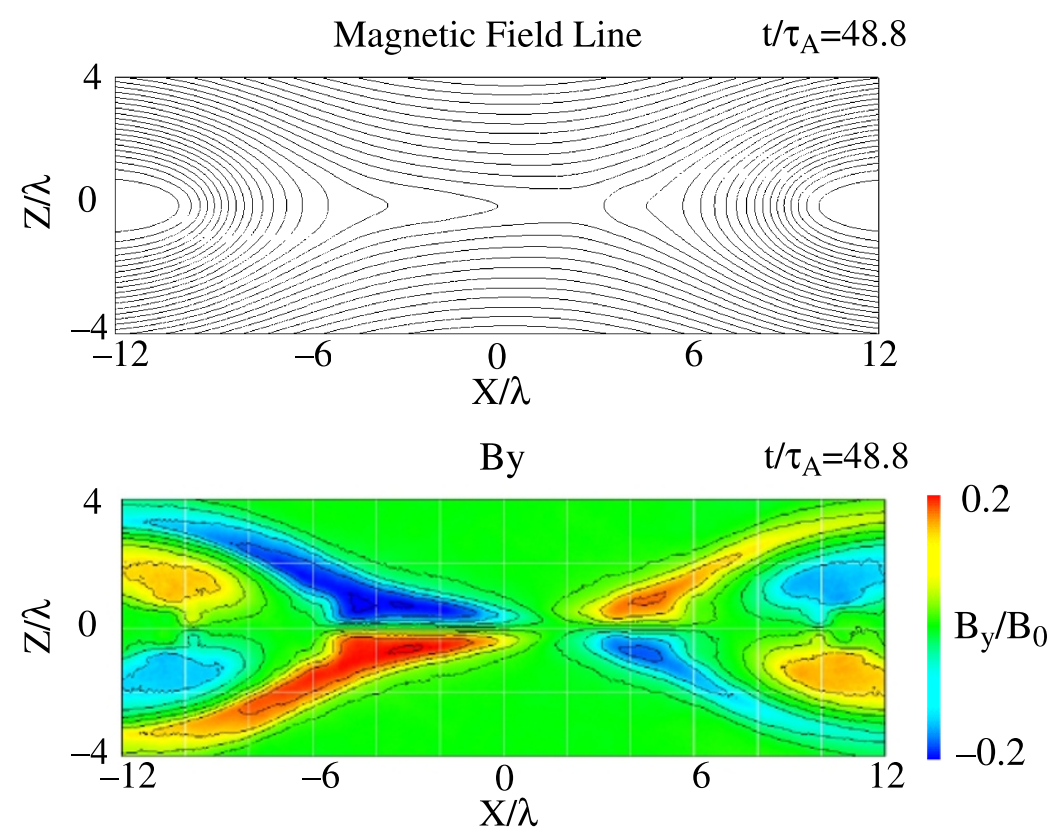

Fig. 4. (a) The magnetic field lines in the $x-z$ plane (top), and (b) the $B_{y}$ magnetic field at $t / \tau_{A}=48.8$. The X-type neutral region is formed around $(X / \lambda, Z / \lambda)=(1,0)$.

thickness of the initial plasma sheet. We assumed the double periodic boundary in the $x$ and $z$ directions. The numerical grid points are $N_{x} \times N_{z}=1600 \times 640$, and the number of total particles is about $2 \times 10^{8}$. The plasma parameters are as follows: Ion inertia length $\left(V_{\mathrm{A}} / \Omega_{\mathrm{i}}\right)$ is $1.15 \lambda$ with 46 grids, and electron inertial length $\left(c / \omega_{\text {pe }}\right)$ is $0.14 \lambda$ with 6 grids. The mass ratio of ions to electrons $m_{i} / m_{e}$ is 64 . The plasma temperatures are set as follows: $T_{\mathrm{i}, \mathrm{ps}} / T_{\mathrm{e}, \mathrm{ps}}=4$, $T_{\mathrm{i}, \text { lobe }} / T_{\mathrm{i}, \mathrm{ps}}=1 / 25$, and $T_{\mathrm{i}, \text { lobe }}=T_{\mathrm{e}, \text { lobe }}$, where the subscript "i" means ion, "e" electron, "ps" the plasma sheet, and "lobe" the lobe, respectively. The initial lobe plasma density $N_{\text {lobe }}$ is set to be $20 \%$ of the plasma sheet density $N_{\text {ps }}$.

The time evolution of reconnection is basically same as other reconnection simulations done in two-dimensional MHD, hybrid and full particle codes so far (e.g., Lottermoser and Scholer, 1997; Shay et al., 1999; Hesse et al., 1999). A snapshot of the magnetic field lines in the reconnection plane and the magnetic field perpendicular to the reconnection plane are shown in Figs. 4(a) and (b), respectively. The X-type neutral point is formed in the center of the simulation box, and the quadrupole magnetic fields are formed in the boundary between the lobe and the plasma sheet. The magnetic fields out of the plane are produced by the Hall electric current effect (Sonnerup, 1979; Terasawa, 1983).

\subsection{Electron distribution functions}

Let us first show the electron distribution functions obtained in our nonlinear simulation. Figure 5 shows the electron distributions together with the "map" of the reconnection structure. The reconnection map shows the magnetic field lines (black lines), plasma density (color contours) and electron flow vectors (red arrows). The two-dimensional slice of the electron distribution functions and their onedimensional cuts are also shown for four typical regions.
The two-dimensional slice is taken from the three-dimensional distribution functions in a plane including the magnetic field vector and the $E \times B$ direction, which is the same format as that of the Geotail distribution functions discussed in Fig. 2. The reddish color corresponds to the higher phase space density region, while the bluish color is the lower density region. The one-dimensional cut attached to the twodimensional slice shows the phase space density along the magnetic field. The logarithmic scales are used for both the color contour of the two-dimensional slice and the onedimensional cut. The velocity is normalized by the electron thermal velocity $V_{e}$ in the initial plasma sheet condition.

The top and left-hand panel is the distribution obtained in the boundary between the lobe and the plasma sheet. We find that the cold population of the reddish color region is flowing along the magnetic field and the hot population having a crescent/lima-bean structure is flowing in the opposite direction against the cold component. The temperature for the cold component is almost same as that in the lobe electron plasma. Since the magnetic field $B_{x}$ is positive in the northern lobe region of $Z>0$, the cold and the hot components are respectively flowing toward and away from the X-type region. This distribution is quite similar to the "shifted-football" distribution observed by Geotail in Fig. 2. From both the electron flow vectors and the magnetic field $B_{y}$ shown in Fig. 4, we find that the cold electrons flowing toward the X-point region are the main carrier of the Hall electric current in the boundary region.

The top and right-hand panel shows the electron distribution function obtained in the plasma sheet side of the "shifted-football" region. The distribution consists of the flat-topped electrons in the lower energy and the suprathermal electrons. The low energy population is characterized by the distribution having a lager parallel temperature than the perpendicular temperature, and the high-energy part is 


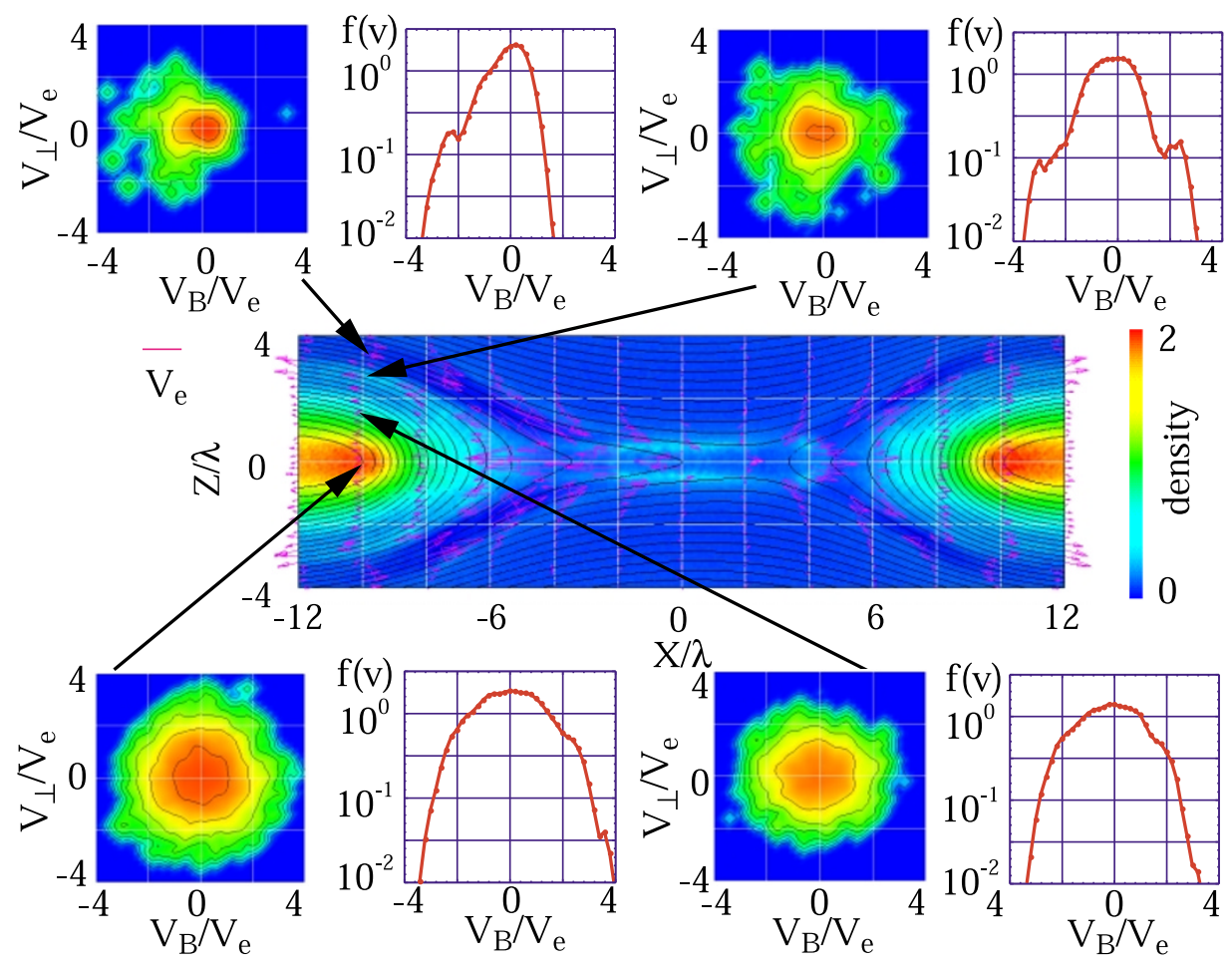

Fig. 5. The electron distribution functions obtained by our particle-in-cell simulation. The reconnection structure is also shown as reference. The magnetic field lines (black), the electron flow vector (red), and the color contour of the plasma density. The format of the electron distribution functions are the same as that of the observations in Fig. 2. The location of the distribution functions are indicated by the black arrow in the map of reconnection.

almost isotropic. We think that this distribution corresponds to the "football" distribution observed by Geotail in Fig. 2.

The bottom and right-hand distribution is obtained in further downstream region, and shows that the electrons are almost thermalized and become isotropic. The parallel temperature is slightly higher than the perpendicular temperature, which probably suggests that the distribution has a memory of the football distribution. Since the low energy part shows slightly a flat distribution, we can compare this with the "truncated-cone" observed in Fig. 2. The bottom, left-hand distribution shows the hot and Maxwellian-like distribution obtained in the central plasma sheet. The parallel and the perpendicular temperatures are almost same, and the temperature is higher than that of the truncated-cone distribution. We think that the distribution may correspond to the Maxwellian distribution discussed in Fig. 2.

It seems that the above numerical simulation demonstrates successfully the evolution of the electron distribution functions observed by Geotail. Let us discuss in details the kinetic aspects of the electron distribution function below.

\subsection{Electron PSBL beams}

We study first how and where the electron crescent beams in the shifted-football are produced. We think that this beam is produced by the Speiser motion near the X-type region (Speiser, 1965), and that the process of the electron crescent beam is same as that of the PSBL ion beams. To confirm this hypothesis, we first pick up only the high speed electrons with $V_{B} / V_{e}<-1$ from the shifted-football distribution function in Fig. 5, and trace the position of particles backward in time. The top panel in Fig. 6 shows the positions of the electrons in four different times of $t / \tau_{A}=48.8$
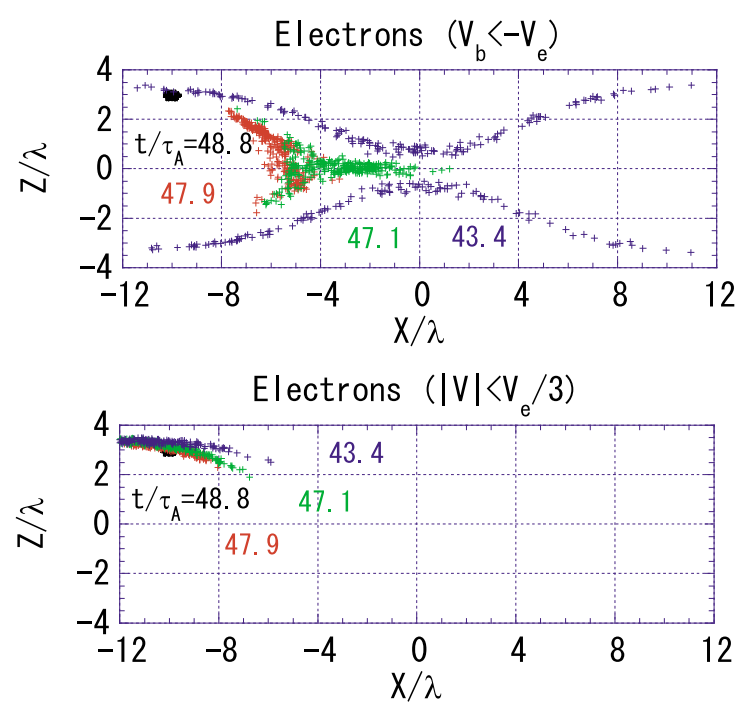

Fig. 6. Position of the high speed electrons (top) and the low energy electrons (bottom) are traced backward in time, respectively.

(black), 47.9 (red), 47.1 (green) and 43.4 (blue). At $t / \tau_{A}=$ 43.4 (blue), the electrons are located outside the separatrix, namely in the lobe magnetic field lines. At $t / \tau_{A}=47.1$ (green), they enter into the plasma sheet. We also found that the electrons gain their energy when they traverse the X-type region by analyzing the relationship between the particles' energy and their positions (not shown here).

Near the X-type region where the ions are unmagnetized but the electrons are still magnetized, the electron motion is 
basically described by the $E \times B$ drift motion, and the electron flow velocity can be expressed by $v_{\text {ele }}=c E_{y} / B_{z}$. For simplicity we may assume that the reconnecting magnetic field $B_{z}$ is approximated by $B_{z}(x)=\left(x / \Delta_{\text {ion }}\right) B_{z}\left(\Delta_{\text {ion }}\right)$ near the X-type region, where $\Delta_{\text {ion }}$ is the size of ion unmagnetized region. Since both ion and electron velocities outside the ion unmagnetized region should be the Alfven velocity $\left(V_{\mathrm{A}}\right)$, we have the relationship of $V_{\mathrm{A}}=v_{\text {ele }}\left(\Delta_{\text {ion }}\right)=$ $c E_{y}\left(\Delta_{\text {ion }}\right) / B_{z}\left(\Delta_{\text {ion }}\right)$. Assuming that the electric field $E_{y}$ is almost constant in the reconnection region, we get

$$
\begin{aligned}
v_{\text {ele }}\left(\Delta_{\text {ele }}\right) & =V_{A} \frac{B_{z}\left(\Delta_{\text {ion }}\right)}{B_{z}\left(\Delta_{\text {ele }}\right)}=V_{A} \frac{\Delta_{\text {ion }}}{\Delta_{\text {ele }}} \simeq V_{A} \frac{c / \omega_{\text {pi }}}{c / \omega_{\text {pe }}} \\
& =V_{A}\left(\frac{m_{\text {ion }}}{m_{\text {ele }}}\right)^{1 / 2} .
\end{aligned}
$$

We find that the electron flow velocity reaches up to the electron Alfven velocity $\left(V_{\mathrm{A}, \mathrm{e}}\right)$ defined by $V_{\mathrm{A}} \sqrt{m_{\text {ion }} / m_{\text {ele }}}=$ $B / \sqrt{4 \pi m_{\text {ele }} n}$. By assuming that the ion temperature in the plasma sheet is almost same as the electron one, we get $V_{\mathrm{A}, \mathrm{e}} \approx V_{\mathrm{e}}$. We find that the agreement of this estimation and the beam speed in Fig. 5 is good. We can conclude that the crescent population of the shifted-football is the counterpart of the PSBL ion beams, and we call this as the PSBL electron beams.

The trace of the particle positions for the cold component of the shifted-football is shown in the bottom panel in Fig. 6. We select the electrons which satisfy the condition of $|V| / V_{e}<1 / 3$ from the distribution at $t / \tau_{A}=48.8$ in Fig. 5. We find that the cold core population is just convected from the lobe to the boundary region without crossing over the X-type region. They are slightly heated in the Hall current layer.

\subsection{Flat-topped electrons in football}

The football electron distribution function can be found in the downstream region of the shifted-football distribution in both our simulation result and the Geotail observations. There are two important signatures: one is the flat-topped distribution in the low energy population, and the other is the almost isotropic high-energy population. We think that two different processes are occurring in the football region.

By analyzing the positions of the low energy electrons backward in time, we find the behavior of the cold electrons is almost same as that for the cold electrons of the shifted-football, namely the low energy electrons are convected from the lobe toward the plasma sheet by the $E \times B$ drift motion (not shown here). The process to produce the flat-topped electron distribution in the lower energy range is as follows: Since the electron velocity reached up to the electron Alfven velocity which is of the order of the electron thermal velocity in the electron PSBL beam region, we may expect two-stream instability between the velocity difference of ions and electrons (e.g., Shimada and Hoshino, 2000) and/or bump-on tail instability of electron (e.g., Omura et al., 1994). Note that the ion thermal/PSBL ion beam speed is of the order of the Alfven velocity, which is much smaller than the electron Alfven velocity. The broadband electrostatic waves which frequencies are lower than the plasma frequency could be excited. Therefore, those waves could effectively scatter electrons along the magnetic field line, and could form the flat-topped distribution in the low energy range.

\subsection{Wave spectra in reconnection region}

In order to check whether or not such electrostatic waves are generated, we show the wave spectra obtained in eight typical reconnection regions in Fig. 7. The vertical axis is the wave power of $E_{x}^{2}+E_{z}^{2}$ and the horizontal axis is the wave frequency normalized by the electron plasma frequency defined by the initial plasma sheet density. The wave power is normalized by $\left(B_{0} V_{A} / c\right)^{2}$. The time interval is $48.8<t / \tau_{A}<51.7$. In this time interval, the magnetic pile-up region moves from $X / \lambda \sim \pm 9$ to $X / \lambda \sim \pm 11$. For each panel, the dashed line is the wave spectrum at $Z / \lambda=2$ and the solid line is obtained at the neutral sheet of $Z / \lambda=0$. The locations of $X$ are respectively $X / \lambda=-9$ for the left and top panel, $X / \lambda=-7$ for the right and top panel, $X / \lambda=-6$ for the left and bottom panel, and $X / \lambda=-5$ for the right and bottom panel. The lower-hybrid frequency calculated from the lobe magnetic field is $\omega_{\mathrm{LH}} / \omega_{\mathrm{pe}}=0.029$. We observe the enhancement of the Langmuir waves for the most of cases, and in the higher frequency range the wave power decreases. The bump of the Langmuir wave well corresponds to the local plasma frequency.

At $(X / \lambda, Z / \lambda)=(-6,2)$ where the strong electron flow toward the $X$-type region are observed, the wave spectrum has a sharp peak around the local plasma frequency of $\omega / \omega_{\mathrm{pe}} \sim 0.2$. In this region, we can observe the shiftedfootball, and we think that the peak of the Langmuir waves may be generated by the electron PSBL beams. At $(X / \lambda, Z / \lambda)=(-7,2)$ where the electron flow directs outward (see also Fig. 5) and where the football electron distribution function is observed, the wave spectrum has a significant power in the lower frequency range. These waves may be excited by the nonlinear stage of the two-stream instabilities (e.g., Davidson et al., 1970; Omura et al., 1994). The waves are mainly generated along the magnetic field direction. Under these waves from the lower frequency to the plasma frequency, we think that the electrons can be effectively scattered in the velocity space, and the parallel electron temperature will become higher that the perpendicular one.

\subsection{Hot populations in football, truncated-cone and Maxwellian}

The next question is the origin of the hot and isotropic population of the football distribution. To understand the mechanism producing the isotropic and energetic electron, we again traced the position of the high-energy electrons backward in time. We found two processes are simultaneously occurring: 1) One is the similar process to the PSBL electron beams. The electrons gain first their energy when crossing the X-type region, and are ejected along the magnetic field. However, since the mirror ratio of the reconnecting magnetic flux tube confining the football type electrons is largely different between the X-type region and the top of the magnetic island, some energetic particles do the bouncing motion and cross several times near the X-type region where the electrons gain the energy from the electric field $E_{y}$. We think that such a multiple crossing of the neutral sheet is important for the energization of particles. If the electric field $E_{y}$ is uniform under the time stationary state 

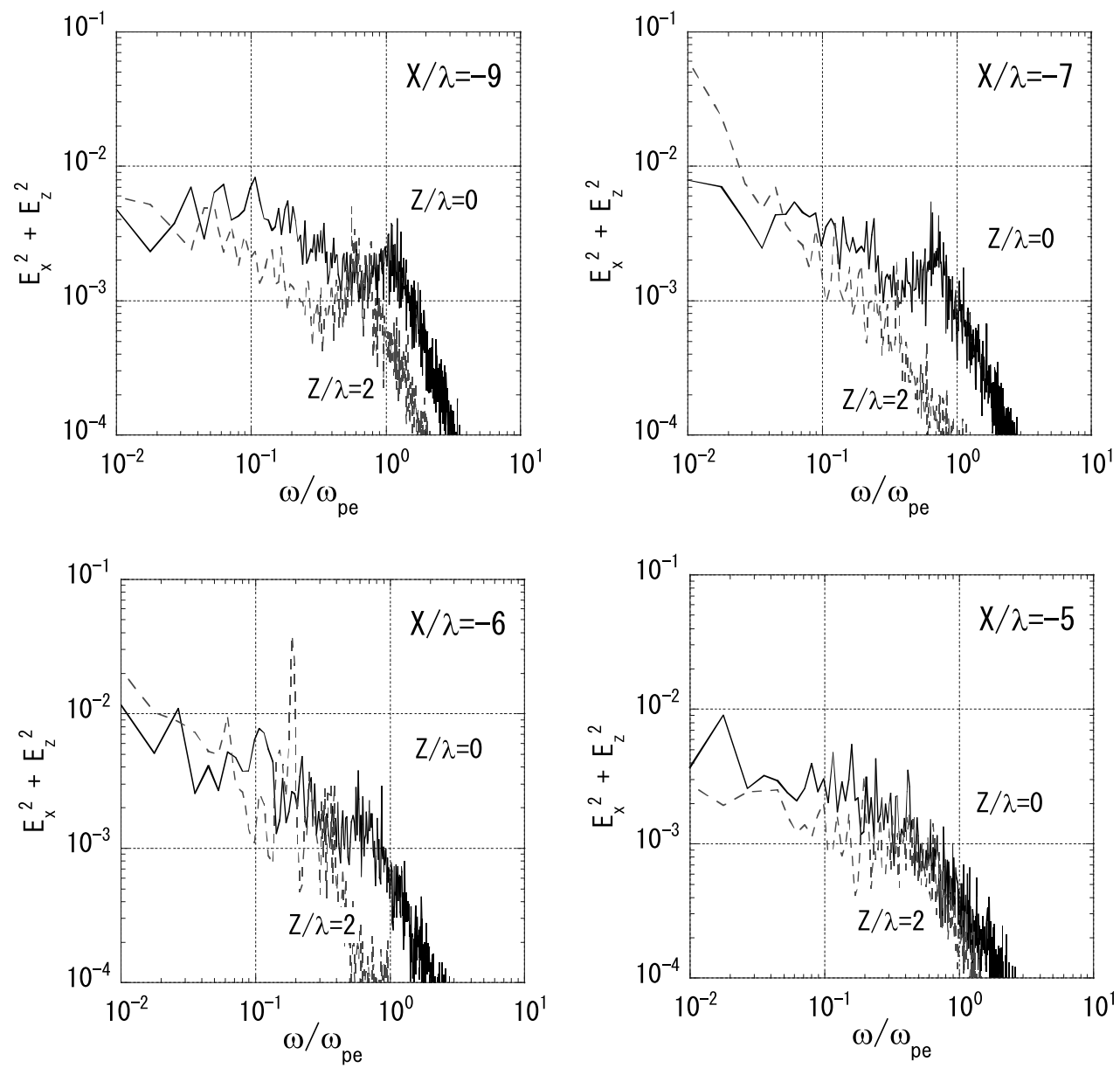

Fig. 7. Wave spectra for the electric field fluctuations of $E_{x}^{2}+E_{z}^{2}$ are shown for eight different positions in reconnection.

$\partial / \partial t=0$, the energy gain of the magnetized electron in the plasma sheet is compensated by the energy loss in the boundary due to $\nabla B$ drift motion. In the time evolutional state of reconnection, the reconnection $E_{y}$ electric field is not uniform. Furthermore, energetic electrons are not magnetized near the X-type region. 2) The other is the trapping effect inside the O-type point. In our simulation, the boundary condition in the $x$ direction is periodic, and the particles that exit through the right (left) hand boundary can enter from the left (right) hand boundary. In the football regions, we found that the energetic particles are trapped around the magnetic island, and they are rolling along the magnetic field line.

In the truncated-cone distribution, the suprathermal electrons are not significantly different from that of the football one, while the lower energy particles become almost isotropic. Inside the magnetic island, we observe the hot Maxwellian-like distribution, probably heated by the magnetic compression due to the pile-up of the reconnecting magnetic field lines.

\section{Discussion and Conclusions}

We study the electron heating process in the magnetic reconnection region by using the particle-in-cell simulation and the Geotail observation. We first found that nonMaxwellian electron distribution functions observed in as- sociation with magnetic reconnection can be classified into four types, and that those distributions have a memory of plasma heating. In the boundary between the lobe and the plasma sheet where the Hall electric current is enhanced during magnetic reconnection, we found the bi-streaming distribution with the cold population flowing toward the X-type region and the hot beam population escaping from the Xtype region. We find that the electron beam speed reaches up to the electron Alfven velocity, and the two-stream instability is switched on. We confirmed from the wave spectra that the broadband waves are excited from the lower-hybrid frequency to the plasma frequency. We have not yet specified the nature of the instability in detail, but such waves could be explained by the nonlinear process of a strong two-stream instability. We discussed that those waves are responsible for the evolution of the electron distribution functions.

Several other important issues still remain resolved. Although the football distribution can be explained by the wave scattering process of the Hall current induced waves, we think that the wave-particle scattering with the lower-hybrid drift (LHD) waves excited by the dawn-dusk electric current $J_{y}$ in the boundary layer (Huba et al., 1978; Shinohara et al., 1998 ) is another important candidate for the formation of the football distribution. The LHD waves propagating obliquely along the magnetic field line and the nonlinear wave-wave couplings can accelerate hot electrons up to a few $\mathrm{keV}$. This 
process is demonstrated by the two-dimensional particle-incell simulation by Shinohara and Hoshino (1999).

In the shifted-football distribution, we presented the distribution having both the cold electron population flowing into the X-type region and the hot electron population flowing out along the magnetic field line from the X-type region. In the lobe side region of the shifted-football, we can find the distribution with only the cold population flowing into the X-type. Therefore, we think that the acceleration region to produce the hot electron beam exists downstream than the cold electron inflowing region. This will be important to modeling the electron magnetic diffusion region around the $\mathrm{X}$-type region.

We did not discuss much on the energy spectra for the high energy population, but we find the non-thermal high energy electrons during the hot electron event discussed in Fig. 2. Up to $\sim 20 \mathrm{keV}$, the distribution can be well approximated by the thermal Maxwellian with $3 \mathrm{keV}$ temperature, and the phase space density of electron is enhanced from the Maxwellian level in the higher energy range of $>20 \mathrm{keV}$. In our simulation result, we confirmed the similar behavior of the high-energy electrons. The study of the nonthermal behavior will be discussed in another paper (Hoshino et al., 2001).

Acknowledgments. The authors have enjoyed useful discussions with Y. Kojima, T. Terasawa, T. Nagai, and A. Nishida.

\section{References}

Baker, D. N. and E. C. Stone, Observations of energetic electrons (E $\geq 200$ $\mathrm{keV})$ in the Earth's magnetotail: plasma sheet and fireball observations, J. Geophys. Res., 82, 1532-1546, 1977.

Davidson, R. C., N. A. Krall, K. Papadopoulos, and R. Shanny, Phys. Rev. Lett., 24, 579-582, 1970.

Feldman, W. C., R. L. Tokar, J. Birm, E. W. Hones, Jr., S. J. Bame, and C. T. Russell, Structure of a slow mode shock observed in the plasma sheet boundary layer, J. Geophys. Res., 92, 83-94, 1987.

Fujimoto, M., M. S. Nakamura, I. Shinohara, T. Nagai, T. Mukai, Y. Saito, T. Yamamoto, and S. Kokubun, Observations of Earthward streaming electrons at the trailing boundary of a plasmoid, Geophys. Res. Lett., 24, 2893-2896, 1997.

Harris, E. G., On a plasma sheath separating regions of oppositely directed magnetic field, Nuovo Cimento, 23, 115, 1962.

Hesse, M., K. Schindler, J. Birn, and M. Kuzunetsova, The diffusion region in collisionless magnetic reconnection, Phys. Plasmas, 6, 1781-1795, 1999.

Hoshino, M., Forced magnetic reconnection in a plasma sheet with localized resistivity profile excited by lower hybrid drift type instability, $J$. Geophys. Res., 96, 11555-11567, 1991.

Hoshino, M., Kinetic Ion Behavior in Magnetic Reconnection Region, AGU monogr., 105, edited by A. Nishida, D. N. Baker, and S. W. H. Cowley, pp. 153-166, 1998.

Hoshino, M., T. Mukai, A. Nishida, T. Yamamoto, and S. Kokubun, Ion dy- namics in magnetic reconnection: Comparisons between numerical simulations and Geotail observations, J. Geophys. Res., 4509-4530, 1998.

Hoshino, M., T. Mukai, T. Terasawa, and I. Shinohara, Suprathermal electron acceleration in magnetic reconnection, J. Geophys. Res., 2001 (in press).

Huba, J. D., N. T. Gladd, and K. Papadopoulos, Lower-hybrid-drift wave turbulence in the distant magnetotail, J. Geophys. Res., 83, 5217-5226, 1978

Lottermoser, R.-F. and M. Scholer, Magnetic reconnection in two-dimensional magnetohydrodynamic and Hall-magnetohydrodynamic, J. Geophys. Res., 102, 4875-4892, 1997.

Mukai, T., S. Machida, Y. Saito, M. Hirahara, T. Terasawa, N. Kaya, T. Obara, M. Ejiri, and A. Nishida, The low energy particle (LEP) experiment onboard the GEOTAIL satellite, J. Geomag. Geoelectr., 46, 669692, 1994.

Nagai, T., I. Shinohara, M. Fujimoto, Y. Saito, M. Hoshino, S. Machida, and T. Mukai, Geotail observations of the Hall current system: Evidence of magnetic reconnection at the kinetic level, J. Geophys. Res., 2001 (in press).

Nishida, A., The Earth's dynamical magnetotail, Space Sci. Rev., 3-4, 507577, 2000.

Omura, Y., H. Kojima, and H. Matsumoto, Computer simulation of electrostatic solitary waves - a nonlinear model of broad-band electrostatic noise, Geophys. Res. Lett., 2923-2936, 1994.

Petschek, H. E., Magnetic field annihilation, NASA-SP-50, 425-439, 1964 Richardson, I. G., S. W. H. Cowley, E. W. Hones, Jr., and S. J. Bame, Plasmoid associated energetic ion bursts in the deep geomagnetic tail: properties of plasmoids and the post-plasmoid plasma sheet, J. Geophys. Res., 92, 9997-10013, 1987.

Saito, Y., T. Mukai, T. Terasawa, A. Nishida, S. Machida, S. Kokubun, and T. Yamamoto, Foreshock structure of the slow-mode shocks in the Earth's magnetotail, J. Geophys. Res., 101, 13267-13274, 1996.

Shay, M. A., J. F. Drake, and B. N. Rogers, The scaling of collisionless, magnetic reconnection for large systems, Geophys. Res. Lett., 26, 2164 2166, 1999

Shimada, N. and M. Hoshino, Strong electron acceleration at high Mach number shock waves: Simulation study of electron dynamics, Astrophys. $J .$, 543, 67-71, 2000.

Shinohara, I. and M. Hoshino, Electron heating process of the lower hybrid drift instability, Adv. Space Res., 24, 43-46, 1999.

Shinohara, I., T. Nagai, M. Fujimoto, T. Terasawa, T. Mukai, K. Tsuruda, and T. Yamamoto, Low-frequency electromagnetic turbulence observed near the substorm onset site, J. Geophys. Res., 103, 20365-20388, 1998.

Sonnerup, B. U. Ö., Magnetic field reconnection, in Solar System Plasma Physics, vol. III, edited by L. T. Lanzerotti, C. F. Kennel, and E. N. Parker, pp. 45-108, North-Holland, New York, 1979.

Speiser, T. W., Particle trajectories in model current sheet 1, Analytical solution, J. Geophys. Res., 70, 4219, 1965.

Terasawa, T., Hall current effect on tearing mode instability, Geophys. Res. Lett., 10, 475-478, 1983.

Terasawa, T. and A. Nishida, Simultaneous observations of relativistic electron bursts and neutral-line signatures in the magnetotail, Planet. Space Sci., 24, 855-866, 1976.

Yamada, M., Review of the recent controlled experiments for study of local reconnection physics, Earth Planets Space, 53, this issue, 509-519, 2001

M. Hoshino (e-mail: hoshino@eps.s.u-tokyo.ac.jp), K. Hiraide, and T. Mukai 